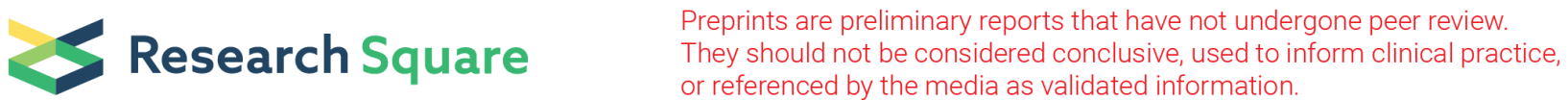

\section{Study on the Mechanism of Tiaogan Yunpi Decoction in Treating Diarrhea-Irritable Bowel Syndrome Based on Network Pharmacology}

Jing Yan

Nanjing University of chinese medicine

Fei Ge

Haian Hospital of Traditional Chinese Medicine

Zhi-wei Miao

Zhangjiagang Hospital of TCM

Li-na Liu

Affiliated Hospital of Nanjing University of Chinese Medicine: Jiangsu Province Academy of Traditional Chinese Medicine

Jun Lu

Affiliated Hoapital of Nanjing University of chinese medicine

Feng Liu

Pukou Hospital of chinese medicine

Wen-bin Shang

Nanjing University of chinese medicine

Juan Zhao

Nanjing University of chinese medicine

Xi-zhong Yu

Nnajing University of chinese medicine

\section{Zhi-guang Sun}

Nanjing University of Chinese Medicine

\section{Li-qin Ning}

Affiliated Hospital of Nanjing University of chinese medicine

Dong-xue Lu (D1606388689@qq.com)

Nanjing University of Chinese Medicine

\section{Research}

Keywords: Tiaogan Yunpi Decoction, Diarrhea-irritable bowel syndrome, network pharmacology, molecular mechanism 
Posted Date: October 16th, 2020

DOl: https://doi.org/10.21203/rs.3.rs-90752/v1

License: (c) (i) This work is licensed under a Creative Commons Attribution 4.0 International License. Read Full License 


\section{Abstract}

Tiaogan Yunpi Decoction (TGYPD) is a clinical experience commonly used by tutors to treat diarrheairritable bowel syndrome (D-IBS); it has been commonly employed to treat ulcerative colitis and chemotherapy-induced intestinal mucositis. However, the mechanism of TGYPD in D-IBS treatment remains unclear. In the present study, the potential mechanism of TGYPD for irritable bowel syndrome was tested by network pharmacology combined with the IBS rat model. On the whole, 56 active ingredients were screened out, and 238 assessed targets were identified; 1934 known disease targets regarding the occurrence and development of irritable bowel syndrome were successfully searched from the disease database. $\mathrm{GO}$ biological processes primarily impact cytokine receptor binding, transcription factor activity, cytokine activity, antioxidant activity, biosynthesis regulation, cell cycle regulation and other cellular active sites of irritable bowel syndrome. Besides, the mentioned processes are involved in AGE- RAGE signaling pathway, TNF and IL-17 signaling pathway, Toll-like receptor (TLRs) signaling pathway, multiple cancer signaling pathways, and viral key signaling pathways of infection, hepatitis and endocrine resistance. As reported by the protein interaction network (PPI), IL-6, CXCL8, VEGFR, JUN, MAPK3 and AKT1 are likely to act as the critical targets for TGYPD to treat IBS. Moreover, in the model of IBS-D rats, TGYPD is capable of significantly reducing stool Bristol type and AWR scores, as well as effectively decreasing TNF-aand IFN- $y$. As revealed from colon pathological section, TGYPD can relieve intestinal damage and mitigate intestinal mucosal immune inflammation. As suggested from the results of the Western blotting assay, TGYPD is capable of suppressing the expression of TLR4-MYD88-NF-kB signaling pathway in intestines. In brief, the results achieved in this study suggest that TGYPD can significantly mitigate immune inflammation and protect against intestinal mucosal barrier in the intestines of the IBS-D rat model. This study provides novel insights that can be referenced by other TCM studies.

\section{Introduction}

Diarrhea irritable bowel syndrome (D-IBS) refers to a general clinical gastrointestinal disease. In the absence of identifiable inflammation, structural or metabolic abnormalities, abdominal pain, urgency, bloating, as well as water samples ${ }^{[1,2]}$, D-IBS is considered one of the gastrointestinal disorders most commonly identified by gastroenterologists and residents. Moreover, IBS imposes a significant economic burden on medical resources and causes the quality of life of patients to decline noticeably ${ }^{[3]}$. Traditional Chinese medicine (TCM) has been employed in Asia for over 2,000 years. Traditional Chinese medicine acts as one of the most popular forms of complementary and alternative medicine in China; it tends to be accepted by non-Chinese medicine practitioners for its prominent efficacy, rich resources, as well as low toxicity. Chinese medicine and its preparations have been extensively employed to prevent and treat diseases worldwide ${ }^{[4]}$. In the clinical treatment of D-IBS, numerous traditional Chinese medicine formulas have been employed alone or as adjuvants for conventional anti-D-IBS drugs. Thus far, to treat IBS and

relevant diseases, Yiqi Jianpi prescriptions (e.g., Sijunzi Decoction ${ }^{[5]}$, Lizhong Pill ${ }^{[6]}$, Xianglian Pill ${ }^{[7]}$, 
Tongxie Yaofang ${ }^{[8]}$ and Jianpi Shugan method ${ }^{[9]}$ ) have been suggested with protective intestinal mucosa and anti-inflammatory effects.

TGYPD is composed of Tongxie Yaofang, Sijunzi Decoction, Lizhong Decoction, as well as Xianglian Pill. It exploits Codonopsis, Stir-fried Atractylodes and Poria, nourishing qi and strengthening the spleen, artillery ginger, nutmeg warming the spleen yang, windproof, tangerine peel, woody fragrance for the regulation of the liver-Qi, Baiji, papaya softening and converging the liver, Sichuan coptis clearing heat and damp, Chao Jianqu dispels facilitate transport process. The combination of ShuYun, rigidity and softness, Wenzhong Zuoqing, can regulate liver and spleen, considering both specimens. The active ingredients of its composition can inhibit visceral sensitivity ${ }^{[10]}$ and regulate gastrointestinal hormones [11]; they have been extensively used to treat diarrhea. By regulating the intestinal flora, one existing study ${ }^{\text {[9] }}$ suggested that Yiqi Jianpi Chinese medicine is capable of elevating the proportion and promoting the distribution of intestinal probiotics, as well as regulating the flora metabolites to protect the intestinal mucosa and treat diarrhea. As reported by Zhuo Bingfan ${ }^{[12]}$ et al., based on the brain-gut axis, the components of TGYPD can mitigate the symptoms of IBS through the regulation of the secretion of brain-gut peptides. He Ying ${ }^{[13]}$ indicated that the flavor of Tongxie Yaofang can reduce the intestinal mucosa by regulating intestinal immunity permeability to protect the intestinal mucosa, as an attempt to treat irritable bowel syndrome. However, the treatment of irritable bowel syndrome from liver and spleen has not been conclusively explained.

TCM refers to a sophisticated system with multiple targets and synergistic or antagonistic interactions among its components ${ }^{[14]}$. The integrity of the whole human body is stressed in the theory of TCM, which is inconsistent with western medicine of "one target, one drug". For its complexity in composition, conventional pharmacological approaches to experimentally identify the unique action of mechanism may not apply to TCM study. Along with the rapid development of bioinformatics, the emerging network pharmacology complies with big databases and has become an effective tool to specifically characterize the action mechanisms of complicated drug system from the molecular level to the pathway level ${ }^{[15]}$. Network pharmacology is consistent with the critical ideas of the holistic philosophy of $\operatorname{TCM}^{[16]}$. As an advanced technology, network pharmacology has acted as a promising method for Chinese medicine

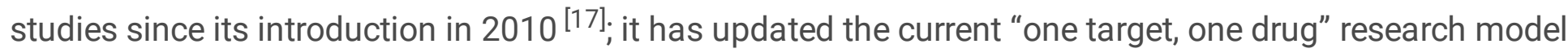
to a novel "multi-component, multi-target" research model. By presenting a specific compound-target and target-pathway network, the rationality and compatibility of traditional Chinese medicine can be more effectively assessed. It has been extensively applied in the mechanism study on TCM to treat complex diseases (e.g., cancer, asthma and cardiovascular disorders). Accordingly, the computational tools and resources were employed to analyze the pharmacological network of TGYPD on IBS, as an attempt to assess active compounds and potential protein targets, as well as pathways. Moreover, animal experiments were performed to verify the potential mechanism of TGYPD for treating IBS based on network pharmacology. The specific flowchart of this study is illustrated in Fig. 1.

\section{Materials And Method}


1 Screening of candidate components based on network pharmacology TGYPD

TCMSP (http://tcmspw.com/) was adopted to retrieve major active ingredients of 12 types of Chinese herbal medicines (i.e., Codonopsis, Fried Atractylodes, Poria, Artichoke Ginger, Nutmeg, Windproof, tangerine peel, woody fragrance, Paeonia lactiflora, papaya, Sichuan coptis and Chaojianqu) ${ }^{[17]}$.

2 Screening strategies for active ingredients of TGYPD

Oral Chinese medicine should address the difficulties attributed to the absorption, distribution, metabolism and excretion before entering the blood to be the active ingredient. In ADME, oral bioavailability refers to one of the critical pharmacokinetic parameters ${ }^{[18]}$. On the whole, a high $O B$ value critically determines the drug similarity index $(D L)$ of an active substance. Components with an $O B \geq$ $30 \%$ are considered with higher $\mathrm{OB}$ values. As a qualitative concept in drug design to assess the drug-like properties of molecules ${ }^{[19]}$, the DL index can be adopted to efficiently screen active substances; it reaches 0.18 on average. Ingredients with a DL index of 0.18 are considered exhibiting higher drug-like properties. Thus, in this study, the compounds that have TGYPD supplemented by $\mathrm{OB} \geq 30 \%$ and $\mathrm{DL} \geq 0.18$ were taken as the active substances.

\section{Drug target prediction of TGYPD}

The protein targets of the active substances of TGYPD were acquired according to the TCMSP and TCMID (http://www.megabionet.org/tcmid/) databases.

4 Collection of target genes for D-IBS

The therapeutic target database (TTD, http://bidd.Nus.edu.sg / group / cjttd /), GeneCards (https://www.genecards.org/) database, and the comparative toxicogenomics database (CTD, http: / / ctdbase. org /) were selected to collect corresponding targets for Irritable bowel syndrome.

5 TGYPD active Ingredient-Target-Disease Target Network Construction

The Merge function in Cytoscape 3.6.0 was adopted, and the 2 results were combined into a medicinal material-active ingredient-key target-disease network diagram. The intersection of the component target map and the disease target map indicates the vital target to treat IBS. Degree refers to the number of nodes connected to other nodes, defining points with Degree $\geq 34$ as vital genes.

6 GO biological process and KEGG pathway enrichment analysis of TGYPD

The gene name of the key target was inputted in the DAVID 6. According to 8 databases (https://david.ncifcrf.gov/) ${ }^{[20]}$ gene list, the "official-gene-symbol" was taken in the gene identifier column, and list of DAVID platforms. The both backgrounds were set to "Homo sapiens" (human), and the analysis tool was "functional annotation tool". Based on $\mathrm{P}<0.01$ and number of targets (Count) $\geq 3$, the top 20 biological processes and KEGG pathways were screened out. 


\section{Experimental verification}

\section{Preparation and Assessment of D-IBS Model Rats}

IBS rat model was prepared by intragastric administration of neonatal maternal separation (NMS) combined with chronic bondage. To be specific, from 2 to 21 days after the birth of SD pups, mothers and infants were separated for $3 \mathrm{~h}$ (9: 00-12: 00) per day; besides, no breastfeeding and no experimental operation was performed for the next 4-6 weeks. At the 7th week, only male SD rats acted as the models (with the effect of estrogen on the model excluded); they were placed in a self-made cage (exhibiting the length of $18 \mathrm{~cm}$, the width of $4 \mathrm{~cm}$, and the height of $4 \mathrm{~cm}$ ); thus, the rats could only move forward and backward and could not turn back and turn left, as well as turning right for $3 \mathrm{~h}$ a day for 10 consecutive days ${ }^{[21]}$. In the normal group, newborns were not separated from mothers and infants. At the 7 th week, male SD rats were intragastrically administered with $1 \mathrm{ml}$ of sterile saline for 3 consecutive days. Lastly, the model was assessed with the following methods (e.g., abdominal wall withdrawal response (AWR), fecal Bristol classification, colonic transport function, and intestinal mucosal histology ${ }^{[22-23]}$ ).

\section{Preparation of TGYPD}

Stir-fried Atractylodes 10g, Paeoniae 10g, Windproof 10g, Chen Pei 6g, Stir-fried Codonopsis 15g, Poria $12 \mathrm{~g}$, Artichoke 5g, Nutmeg 5g, Sichuan coptis $3 \mathrm{~g}$, Alder Fragrant 10g, Papaya $15 \mathrm{~g}$ and Fried Jianqu $15 \mathrm{~g}$ were cooked with the conventional method, concentrated to a liquid supplemented by $1.2 \mathrm{~g}$ of crude drug per milliliter, tuned to $\mathrm{pH} 7.0$, filtered and sterilized after aseptic operation, and then stored in a refrigerator at $4^{\circ} \mathrm{C}$ for subsequent application.

\section{Grouping and administration}

The rats in the model group were randomly divided into 3 groups, 8 in each group: model + normal saline group, model + TGYPD low dose group,model + TGYPD high-dose group. The dosage is calculated based on the body surface area. The normal group was given sterile normal saline by gavage. The gavage volume of each group was $0.1 \mathrm{ml} / 10 \mathrm{~g}$, once a day for 2 weeks.

\section{Abdominal wall withdrawal response (AWR)}

The rats were fasted for $18 \mathrm{~h}$ before the experiment, and they were anesthetized by ether inhalation. An $8 \mathrm{~F}$ catheter with a latex balloon (exhibiting the diameter of $2.5 \mathrm{~cm}$ and the length of $4 \mathrm{~cm}$ ) was inserted into the rectum via the anus; the tail of the balloon was $1 \mathrm{~cm}$ from the anus. The catheter was in the rat's tail. The catheter was connected to the syringe and the manometer through a tree. The rats were placed in a homemade plexiglass observer; when they woke up and adapted to CRD, the dilatation pressures 
reached 20, 40, 60 and $80 \mathrm{mmHg}$, and the pressures lasted for $20 \mathrm{~s}$ at intervals of $4 \mathrm{~min}$. AWR score was acquired based on rat response (Supplementary Material 1)

\section{Defecation numbers}

After the modeling was achieved, a clean filter paper was placed in the cage, and the number of defecation particles were observed in the model group and normal group in 4h (8: 00-12: 00).

\section{Fecal Bristol Grading}

After the treatment, the feces were collected from 20:00 pm to 8:00 the next morning in a metabolic cage on the same day, and they were allowed to drink and feed freely. The number of fecal particles was determined, and the points were recorded according to the Bristol classification (Supplementary Material 2). Besides, the average score was calculated.

\section{Colonic tissue HE staining}

The animals were sacrificed after being anesthetized with $0.3 \%$ sodium pentobarbital, and the distal colon tissues were removed. Furthermore, a part of $10 \%$ formalin was fixed, paraffin was embedded, and conventional HE staining was performed.

\section{ELISA}

The distal colon intestinal tissue homogenates were extracted, and the contents of TNF- $\mathrm{a}$ and IFN- $\gamma$ were measured by complying with the operation of the kit. The supernatant was collected, and the contents of TNF- $a$ and IFN- $y$ were measured following the operation of the kit .

\section{Immunohistochemistry}

The intestinal tissues were treated with the non-biotin two-step method (SP method). To be specific, the paraffin sections were dewaxed into water, and the primary antibody (1:500) was added to the blocking solution and then incubated at $4^{\circ} \mathrm{C}$ overnight, and subsequently rinsed in $0.01 \mathrm{M}$ PBS for $15 \mathrm{~min} \times 3$ times; the sections were blocked with $5 \%$ BSA, $5 \%$ goat serum in PBS for 30 min at ambient temperature, and the secondary antibody (1:500) was added to the blocking solution and incubated at ambient temperature for $3 \mathrm{~h}, 0.01$ Rinse in M PBS for $15 \mathrm{~min} \times 3$ times; the reagent SABC was added and incubated at $37^{\circ} \mathrm{C}$ for $20 \mathrm{~min}$, the color was developed with $\mathrm{DAB}$, the sample was dehydrated with gradient alcohol and covered with xylene after turning transparent.

\section{Western Blot}

The tissues and cells were homogenized, lysed with RIPA buffer mixed with protease inhibitor, and centrifuged ( $12500 / \mathrm{min}, 15 \mathrm{~min}, 4^{\circ} \mathrm{C}$ ). After the protein was quantified, an aliquot of the supernatant supplemented by 50 ug of protein was employed for the separation, electrophoresis, membrane transfer, staining, membrane cutting, decolorization, blocking, and membrane washing processes. The primary 
antibody was diluted and incubated. The sample was shaken overnight at $4^{\circ} \mathrm{C}$. After the secondary antibody was diluted, the shaking process was conducted for $90 \mathrm{~min}$ at $37^{\circ} \mathrm{C}$. ELC fluorescent substrate was added for color development, exposure and pictures collection.

\section{Data analysis}

All data were processed with Graphpad 8.3 statistical software. The measurement data were expressed as $(x \pm s)$. The comparisons between the three groups were drawn with One-way ANOVA. The comparisons between the two groups were conducted by t-tests. Grade indicators Spearman correlation coefficient analysis was analyzed; $P<0.05$ indicated that the difference is of statistical significance.

\section{Results}

\section{Network Pharmacology-Based Analysis}

\section{Tiaogan Yunpi Decoction Bioactive Ingredients}

On the whole, 1271 compounds were produced and then screened in accordance with their ADME parameters. The conditions consisted of $\mathrm{OB} \geq 30 \%$ and $\mathrm{DL} \geq 0.18$. 68 compounds were identified to be eligible, with 14 as Codonopsis, 2 as Atractylodes root, 5 as Poria, 4 as Cannon ginger Species, 4 as nutmeg, 6 as windproof, 4 as tangerine peel, 4 as woody fragrance, 3 as white scallion, 2 as papaya, 7 as Chuanhuanglian, and 13 as fried Jianqu. 12 duplicates were deleted, and 56 biologically active ingredients were overall employed to conduct the subsequent analysis (Table 1)

\section{Tiaogan Yunpi Decoction recognizes D-IBS targets}

321 active ingredient targets were overall obtained from TCMSP and TCMID; they were entered into the UniProt database and converted into the corresponding gene names. On the whole, there were 1685 targets. The duplicate targets were deleted, and the rest 238 targets were acquired. A total of 40831 edges were obtained (Fig. 2). 1945 D-IBS potential targets were identified in TTD and GenCards, and 1934 were obtained after the duplicate targets were eliminated.

\section{Network diagram and analysis of components-targets-D-IBS targets of Tiaogan Yunpi Decoction}

Since traditional Chinese medicine prescriptions exhibit multiple pharmacological activities via multiple targets, it is constructive to study the mechanism of traditional Chinese medicine prescriptions on complex diseases online. The 151 intersection genes of the component target and IBS target drawn by the Wayne diagram were introduced into Cytoscape 3.6.0, and a target network of TGYPD-D-IBS composite target network was built (Fig. 3). Furthermore, a total of 44 nodes and 164 edges were covered.

\section{$4 \mathrm{GO}$ biological process and KEGG enrichment analysis}


The taken 151 vital target genes were mapped to the DAVID platform for relevant biological process entries. The screening process was performed with $P<0.01$ and the number of targets $\geq 3$. Besides, a total of 144 biological functions were obtained. The top 20 results are elucidated as Cytokine receptor binding, nuclear receptor activity, transcription factor, cytokine activity, steroid hormone receptor activity, antioxidant activity, cofactor binding, ubiquitin protein ligase, etc(Fig.4A). To determine the potential pathway of TGYPD for D-IBS, KEGG enrichment analysis was conducted on 151 vital target genes. A total of $160 \mathrm{KEGG}$ pathway enrichment entries were acquired. The first 20 major selections involved the AGERAGE signaling pathway, TNF and IL-17 signaling pathways, Toll-like receptor (TLRs) signaling pathways, multiple cancer signaling pathways, viral infections, hepatitis, Endocrine resistance, etc. (Fig. 4B).

\section{PPI network construction and analysis}

To clarify the interaction between critical target proteins, the 151 vital target genes screened were mapped to the STRING database platform (https // string-db.org /) to build a PPI network model. Besides, the protein type was set to "Homosapiens (Human)", the minimum interaction threshold was set to "medium confidence", and other parameters were left at the default settings. The PPI network model was imported into Cytoscape 3.6.0 software, and the "Network Analysis" function was employed for analysis(Fig.5A). The interaction between vital target proteins was determined given the degree value and the median, and the Hub genes were screened to form a subnetwork of the hub genes. As shown in Fig. 5B, a total of 10 genes of VEGF, CXCL8, IL-6, MAPK8, MAPK3, MMP9, PTGS2, JUN, AKT1 and CASP2 were labeled as hub genes. The mentioned genes may be the vital targets of Tiaogan Yunpi Decoction to treat IBS. Combining GO biological processes and KEGG enrichment analysis, inflammation-related pathways are considered the most meaningful pathways; among the mentioned 10 hub genes, CXCL8, IL-6, VEGF, JUN and others were identified as the relatively high targets. It critically impacted the inflammatory activation and oxidation pathways of cells; it is considered a key marker to treat IBS by TGYPD.

\section{Assessment of the overall efficacy of TGYPD on D-IBS model rats}

To verify the therapeutic effect of TGYPD on D-IBS rats, a D-IBS rat model was built. Compared with the model group, the rats in the TGYPD group and Peifeikang group generally improved their hair, activity and others. $(p<0.001)$ and gained weight $(p<0.01, p<0.001)$ (Fig. $6 A)$. For the number of defecation particles and feces Bristol classification, the TGYPD and Peifeikang groups were significantly reduced, $(p<0.05, p$ $<0.001)$ and $(p<0.01$ and $p<0.001)$ respectively (Fig. $6 \mathrm{~B}-\mathrm{C})$. Under the $20 \mathrm{mmHg}, 40 \mathrm{mmHg}, 60 \mathrm{mmHg}$ balloon pressure, TGYPD combination and Peifeikang group could reduce AWR score, and both groups impact AWR score under $80 \mathrm{mmHg}$ (balloon pressure > 0.05) (Fig. 6D). The colonic pathology exhibited slight organic variations in the colonic mucosal tissue of the model group, whereas there was low-grade inflammation of the intestinal mucosa, including epithelial cell edema, occasionally mild dilatation of submucosal blood vessels, and infiltration of inflammatory cells (e.g., neutrophils and lymphocytes). After the treatment of Tiaogan Yunpi Decoction, no low-grade inflammation was identified in the intestinal mucosa, which demonstrates that TGYPD can mitigate intestinal mucosal inflammation and immune responses (Fig. 7A-B). TGYPD low, high-dose group could significantly inhibit TNF-a levels ( $p$ 
$<0.05)$, whereas they slightly impacted IFN-y levels, revealing that Tiaogan Yunpi Decoction can mitigate low-grade D-IBS inflammation (Fig. 7C-D)

\section{Effect of Tiaogan Yunpi Decoction on inflammatory signaling pathway}

To further verify the effect of TGYPD on D-IBS inflammatory signaling pathway TLRs, the Western Blotting technology was adopted, and the expressions of TLR4, Myd88 and NF-kappbB p65 protein were measured. As revealed from the results, the relative gray values of TLR4, Myd88, and NF-kappbB p65 proteins in the model group were significantly higher than those in the control group, exhibiting significant statistical differences $(P<0.001)$ (Fig. 8A). TGYPD could significantly down-regulate the expression of TLR4, Myd88, and NF-kappbB p65 protein, with statistical differences $(P<0.05, p<0.01, P<0.001)$ (Fig. $8 \mathrm{E})$. As demonstrated from the results of immunohistochemistry, the concentrations of TLR4, Myd88, NFkappbB p65 in the model group were higher than those of the normal group, showing significant differences $(P<0.001)$. However, unlike in the model group, the concentrations of TLR4, Myd88 and NFkappbB p65 markedly decreased in the TGYPD-L and TGYPD-H, respectively(Fig.8B-D).

\section{Discussion}

D-IBS refers to a common gastrointestinal disease in clinic. A variety of factors lead to its recurrence and protracted refractory. As suggested from the Meta ${ }^{[24]}$ analysis, the global incidence of this disease is about $11 \%$. The cost of treating IBS in the United States alone has reached 30 billion US dollars each year, seriously affecting the quality of life of patients and consuming considerable public health resources. Given its clinical characteristics, it pertains to "diarrhea" "abdominal pain" "constipation" and "Depression" and others in the scope of traditional Chinese medicine. Clinically, diarrhea, abdominal pain, constipation, or diarrhea and constipation are alternated, especially when mood varies. The side effects of drug treatment in modern medicine are significant, and most drugs target a single target protein, so the overall treatment effect is limited, while traditional Chinese medicine is composed of multiple compounds, exhibits a wide range of pharmacological activities, and has multiple targets and pathways, which may have therapeutic benefits for D-IBS ${ }^{[25]}$. Clinical studies reported that TGYPD significantly impacts D-IBS, with an effective rate of $91.49 \%$, and slight adverse reactions ${ }^{[26]}$. As revealed from preliminary experimental studies, the mechanism of action is related to the regulation of brain-gut axis, the reduction of brain-gut peptide serotonin (serotonin, $5-\mathrm{HT}$ ), vasoactive intestinal peptide (vasoactive intestinal peptide, VIP), P substance (substance P,SP), the regulation of gastrointestinal motility and the reduction of visceral hypersensitivity ${ }^{[27-28]}$. On the other hand, this characteristic of traditional Chinese medicine will hinder the in-depth study on its underlying mechanism.

Network pharmacology method, integrating system biology and computer technology, presents a direction for the mechanism research of complex Chinese medicine ${ }^{[29]}$. At present, numerous studies have made an effort to adopt network pharmacology to test the intricacy of ingredients, targets, pathways, and mechanisms of action of herb pairs or herbal formulas ${ }^{[30-31]}$. Thus, the network 
pharmacology method was adopted to elucidate the pharmacological mechanism of TGYPD for treating D-IBS.

By network pharmacological analysis, a total of 56 active ingredients were screened out, and 238 assessed targets were identified,1934 known disease targets related to the occurrence and development of irritable bowel syndrome were searched from the disease database. As suggested from research, the main active ingredients of TGYPD were Codonopsis glycosides, Atractylolide III, Flavonoid glycosides, Atractylolide, Xylolactone, Berberin, Hanbain, Coumarin, Orange Cortex, Flavone, Poria, Artemisinin, etc. Modern pharmacological studies indicate that the biologically active substances of TGYPD can act on the immune system, nervous system and digestive system, as well as having anti-inflammatory and antioxidant effects and regulating intestinal flora ${ }^{[10-13]}$. GO biological process and KEGG enrichment analysis reveal that TGYPD for treating IBS primarily involves the participation of multiple biological processes and multiple pathways, especially in terms of receptor activity, transcription factor activity, immune inflammation and apoptosis (Fig.4(a)). As indicated from the KEGG enrichment analysis, the AGE-RAGE signaling pathway, TNF and IL-17 signaling pathways, and Toll-like receptor (TLRs) signaling pathways are all the ways of TGYPD to treat IBS (Fig.4(b)). To be specific, TLRs signal pathway is one of the most common signal pathways of IBS. TLRs receptors are one of the cell transmembrane receptors and pathogen pattern recognition receptors in the innate immune system, primarily involved in the identification of pathogenic microbial products and inflammatory signaling, and critically impacting the homeostasis. It can be expressed on various cells in the intestinal mucosa and mediates the interaction between microorganisms and cells ${ }^{[32]}$. This family includes at least 12 members, of which TLR4 and DIBS are most closely related. Studies have found that ${ }^{[33-36]}$, under normal conditions, and TLR4 are lowexpressed on the colon IEC, which weakens the recognition of intestinal bacteria and is in a "tolerant" state, whereas its expression in D-IBS patients and model animals was significantly higher than that in the normal group.TLR4 refers to the main receptor for gram-negative lipopolysaccharide (LPS) responses [37-38], thereby passing Myeloid differentiation factor 88 (MyD88) -dependent pathway. As a result, NF-KB is ultimately activated, and the IEC is induced to release more pro-inflammatory cytokines tumor necrosis

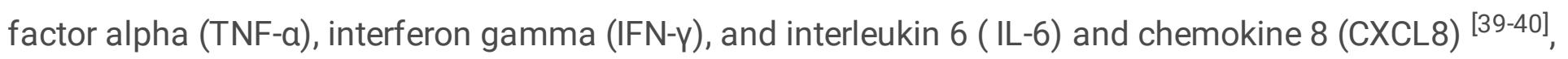
to reconstitute intestinal mucosa tight junction proteins Claudin-1, Occludin-1, ZO-1, and to destroy the intestinal mucosal mechanical barrier ${ }^{[41-42]}$. Existing studies reported that the TLR4/NF-KB signaling pathway is highly expressed in IBS-D rats, the expressions of inflammatory factors IL- 6 and TNF- $a$ are upregulated, the expression of IL-10 is down-regulated, the flora is imbalanced, and the intestinal mucosal mechanical barrier is destroyed ${ }^{[43-45]}$, Berberine, the main active component of Tiaoganyunpi Decoction, is capable of down-regulating the expression of TLR4/NF-KB to regulate the visceral sensitivity and motility abnormalities in D-IBS rats, as an attempt to mitigate symptoms ${ }^{[46]}$ and paeoniflorin ${ }^{[47]}$. The components of Radix Paeoniae Radix ${ }^{[48]}$ can mitigate symptoms (e.g., abdominal pain and diarrhea) by regulating the high sensitivity of the internal organs of D-IBS rats and down-regulating the expressions of inflammatory factors (IL-6, IL-1). 
Accordingly, based on network pharmacology and literature research, this study speculates that TGYPD may be able to treat D-IBS by inhibiting the TLRs signaling pathway and downstream inflammatory factors. However, the relevant experiments are required to support this hypothesis. Thus, this study selected TLRs signaling pathway and downstream inflammatory factors as indicators and revealed the pharmacological mechanism of TGYPD for treating D-IBS by animal experiments. An IBS rat model, which was supplied with TGYPD, was built to confirm its anti-inflammation effect; stool Bristol type, AWR scores was significantly reduced by TGYPD and TNF-, IFN- and MPO decreased as well; colon pathological section implied that TGYPD could relieve intestinal damage and Intestinal mucosal immune inflammation, demonstrating that TGYPD more significantly impacts serum Inflammatory factors and intestinal function.

In the present study, network pharmacology and verification experiments were performed to reveal the potential underlying mechanisms of TGYPD against IBS. Numerous compounds of TGYPD could effectively protect against D-IBS via multiple targets by numerous biological processes and pathways that exhibit different cell components. Moreover, TGYPD may mitigate intestinal damage and intestinal mucosal immune inflammation by down-regulating the expressions of inflammatory factors through the suppression of TLR4 signaling pathway in the intestinal to protect the intestinal mucosal barrier.

\section{Conclusion}

In conclusion, the existing bioinformatic data demonstrated that a network analysis is effectively built to reveal TGYPD-mediated pharmacological biotargets that treat D-IBS, followed by verification experiments. Moreover, the suppression of TLRs signaling pathway and downstream inflammatory factors may be the primary pharmacological mechanisms of TGYPD that treat IBS.

\section{Declarations}

\section{Data Availability}

The data used to support the findings of this study are available from the corresponding author upon request.

\section{Ethical Approval}

Not applicable

\section{Consent for publication}

Authorization and consent publications have been written.

\section{Authors' email addresses and contributions:}

Jing Yan: yanjing@njucm.edu.cn 
Contribution: contributed to the study design, data analysis and interpretation, and writing the manuscript.

Dongxue Lu:1606388689@qq.com

Contribution: contributed to the study design, data analysis and interpretation, and the revision and translation of the article.

Fei Ge:ha9099@163.com

Contribution: contributed to the study design, data analysis and interpretation, and help writing the manuscript.

Li-qin Ning:1623383282@qq.com

Contribution:contributed to the study design, data analysis and interpretation,

Zhiguang Sun and Juan Zhao: pr-zhiguangsun @163.com /zhaojuannj @outlook.com

Contribution: conceived the study question, and guide the writing of articles.

Feng Liu and Xi-zhong Yu:399642091@qq.com/93624680@qq.com

Contribution: undertook data collection and data analysis.

Zhi-wei Miao and Li-na Liu:zjgzy011@njucm.edu.cn/lina_liu@ njucm.edu.cn

Contribution: undertook data collection and data analysis.

Jun Lu and Wen-bin Shang:aztec0403@163.com/wbshang@ njucm.edu.cn

Contribution: undertook data collection and data analysis

\section{Acknowledgement}

Upon the completion of the thesis, I would like to take this opportunity to express my sincere gratitude to all authors $\llbracket$ who were given me important guidance on the thesis. Without their help and encouragement, my thesis would have been impossible. Besides their help with my thesis, they have also given me much advice on the methods of doing research, which is of great value to my future academic life.

\section{Author Disclosure}

All authors have [completed the financial and personal relationships disclosure form] and declare that: (i) we have financial support for study,that is National Natural Science Foundation of CN(81673795,81503536),the Natural Science Foundation of Jiangsu Province [BK20181235] $ه$ Nantong 226 high level talent project; A Project Funded by the Priority Academic Program Development of Jiangsu 
Higher Education Institutions(PAPD);and (ii) there was no conflict of interest in the study;and(iii)there are no other relationships or activities that could appear to have influenced the submitted work.

\section{References}

[1]DiChen,Jie Shen,Weiwei Zhao,et al.Osteoarthritis:toward a comprehensive understanding of pathological mechanism[J].Bone Research,2017,5(01):1-13.

[2]WANG Bin, XING Dan, DONG Shengjie, TIE Ruxiu, ZHANG Zhiqiang, LIN Jianhao, WEI Xiaochun.Prevalence and disease burden of knee osteoarthritis in China: a systematic review[J].Chinese Journal of Evidence-Based Medicine,2018,18(02): 134-142.

[3]SHAKOOR MA,RAHMAN MS,AZAD AK, et al.Effects of isometric quadriceps muscle strengthening exercise on chronic osteoarthritis of the knee[J].Bangladesh Med Res Counc Bull,2010,36 (1):20-22

[4]LI Ning-hua, XUE Qing-yun, ZHANG Yi, ZHANG Yao-nan, WANG Xiao-bin, SHEN Jian, ZHAO Lilian.Epidemiological study on $r$ adiogr aphic knee osteoarthritis in the middle- aged and elderly population in six cities of China [J].The Journal of Practical Medicine, 2008,24(16):2887-2888.

[5]State Technical Supervision Bureau. Standards of the People's Republic of China Standard 1 Terms of Clinical Diagnosis and Treatment of Traditional Chinese Medicine [M]. Beijing: China Standards Press,2000:824.

[6]Li Weixian,Wang Wenxhan囚Liu Yuanyuan,Wang Yiyi.Comparative Study on the Effect of Duhuoshengtang Decoction of Different Processed Products on Adjuvant Arthritis in Rats[J/OL].Acta Chinese Medicine and Pharmacology,2019(05):26-30 [2019-10-29].

[7]Gao Kun, Zhang Yong, Chen Dayu, Yu Weiji, Sun Shufen, Wu Yihong, Lin Zhanpeng, Cao Yafei.Effects of total saponins of Achyranthes bidentata on cytokine profiles of synovial fluid in experimental rabbits with knee osteoarthritis[J].Chinese Journal of Tissue Engineering Research,2019,23(33):5317-5321.

[8]Ma Dujun, Peng Liping, Yu Tian, Cao Yafei, Gao Kun, Xiao Wei, Hu Liekui, Xu Wenming.Influence of Achyranthes bidentata saponins on the repair of cartilage and hypoxia-inducible factor 1 signaling pathway in a rabbit model of osteoarthritis

[J].Chinese Journal of Tissue Engineering Research,2019,23(27):4332-4337.

[9]Ma Dujun,Peng Liping,Cao Yafei,Gao Kun,Wang Lixin,Yu Tian,Xiao Wei,Liao Zhouwei,Hu Liekui.The Experimental Research of Medicated Joint Fluid of Total Saponins of Achyranthes from Experimental Rabbit Model of OA for Cartilage Cell Proliferation and Apoptosis [J].Chinese Journal of Traditional Medical Traumatology \& Orthopedics,2019,27(03):1-5

[10] HOPKINS A. Network pharmacology:the next paradigm in drug discovery[J]. Nature Chemical Biology,2008,4(11):682-690. 
[11]RU J,PENG L,WANG J,et al.TCMSP:a database of systems pharmacology for drug discovery from herbal medicines[J]. J Cheminform,2014,6(1):13.

[12]WU Dan, GAO Yao, XIANG Huan, XING Jie, HAN Yu-mei, QIN Xue-mei, TIAN Jun-sheng.Exploration into mechanism of antidepressant of Bupleuri radix

based on network pharmacology[J].Acta Pharmaceutica Sinica,2018,53(02):210-219.

[13]McAlindon TE, Bannuru RR, Sullivan MC, et al. OARSI guidelines for the nonsurgical management of knee osteoarthritis[J]. Osteoarthr Cartil,2014;22:363-88.

[14]Ma, Y. M., Zhang, X. Z., Su, Z. Z., Li, N., Cao, L., Ding, G., et al. Insight into the molecular mechanism of a herbal injection by integrating network pharmacology and in vitro. J. Ethnopharmacol,2015, 173, 9199.

[15]Chen, Y., Kern, T. S., Kiser, P. D., and Palczewski, K. Eyes on systems pharmacology[J]. Pharmacol. Res,2016,114, 39-41. doi: 10.1016/j.phrs.2016.09.026

[16]Li, S., and Zhang, B. Traditional Chinese medicine network pharmacology: theory, methodology and application. Chin. J. Nat. Med,2013, 11 (2), 110-120.

[17]Ru, J., Li, P., Wang, J., Zhou, W., Li, B., Huang, C., et al. TCMSP: a database of systems pharmacology for drug discovery from herbal medicines[J]. J. Cheminf,2014, 6, 13.

[18]Xu, X., Zhang, W., Huang, C., Li, Y., Yu, H., Wang, Y., et al. A novel chemometric method for the prediction of human oral bioavailability[J]. Int. J. Mol. Sci,2012c, 13 (6), 6964-6982.

[19]Tao, W., Xu, X., Wang, X., Li, B., Wang, Y., Li, Y., et al. Network pharmacology-based prediction of the active ingredients and potential targets of Chinese herbal Radix Curcumae formula for application to cardiovascular Disease[J]. J. Ethnopharmacol,2013,145 (1), 1-10.

[20]Huang da, W., Sherman, B. T., and Lempicki, R. A. Systematic and integrative analysis of large gene lists using DAVID bioinformatics resources[J]. Nat. Protoc,2009,4 (1), 44-57.

[21]YANG cheng,ZHANG sheng-sheng,LI Xiao-ling,WANG Zheng-fang. Establishment and evaluation of rat model of diarrhea predominant irritable bowel syndrome with the method of neonatal maternal separation plus restraint stress[J].Chinese Journal of Integrated Traditional and Western Medicine on Digestion,2015,23(06):377-380

[22]O'Mahony, SM, Marchesi, JR, Scully, P, et al. Early life stress alters behavior, immunity, and microbiota in rats: implications for irritable bowel syndrome and psychiatric illnesses[J]. Biol Psychiatry, 2009, 65(3): 263-67.

[23]Pimentel M, Chatterjee S, Chang C, Low K, Song Y, Liu C, Morales W, Ali L, 
Lezcano S, Conklin J, et al. A new rat model links two contemporary theories in irritable bowel syndrome[J]. Dig Dis Sci,2008, 53:982-989.

[24]Xu D, Gao J, Gillilland M 3rd, et al. Rifaximin alters intestinal bacteria and prevents stress-induced gut inflammation and visceral hyperalgesia in rats. Gastroenterology[J]. 2014, 146(2):484-96e4.

[25]Ma TY, Nguyen D, Bui V, et al. Ethanol modulation of intestinal epithelial tight junction barrier [J] . Am J Physiol. 1999; 276(4 Pt 1):G965-74.

[26]YE Bo,CHEN Jing,NING Li-qin,LIU Chun,CAO Ting-ting.Clinical Study on Tiaogan Yunpi Decoction in Treating Diarrhea-predominant Irritable Bowel Syndrome[J].Journal of Nanjing University of Traditional Chinese Medicine,2013,29(04):314-316.

[27]LIU Chun.Clinical and Experimental Study on Tiaogan Yunpi Decoction in Treating Diarrheapredominant Irritable Bowel Syndrom[J].Nanjing University of Chinese Medicine,2011.

[28]Huo Qingping,Zhang Zhongwei,Li Jinpu,Wang Yuxin,Liang Fang.The Influence of Tiaogan Yunpi Decoction on Regional Encephalic Activation of MR Signal Intensity among Diarrheal-predominant Irritable Bowel Syndrome[J].Journal of Zhejiang University of Traditional Chinese Medicine,2008(05):603605.

[29] R. Z. Zhang, S. J. Yu, H. Bai, K. Ning.TCM-mesh: the database and analytical system for network pharmacology analysis for TCM preparations[J].Scientifific Reports,2018:2821.

[30] J. Zhang, R. Liang, L. Wang, B. Yang.Effffects and mechanisms of Danshen-Shanzha herb-pair for atherosclerosis treatment using network pharmacology and experimental pharmacology[J]Journal of Ethnopharmacology, 2019(229):104-114.

[31] W. Wang, T. Liu, L. Yang, et al.Study on the multi-targets mechanism of triphala on cardio-cerebral vascular diseases based on network pharmacology [J] .Biomedicine \& Pharmacotherapy,2019(116): 108994.

[32] LUKIC I K,HUMPERT P M,NAWROTH P P,et al. The RAGE pathway \activation and perpetuation in the pathogenesis of diabetic neuropathy[J]. Annals of the New York Academy of Sciences,2010,1126(1):7680

[33] XU X X,ZHANG X H,DIAO Y,et al.Achyranthes bidentate saponins protect rat articular chondrocytes against interleukin-1 $\beta$ - induced inflammation and apoptosis in vitro [J]. The Kaohsiung journal of medical sciences,2017,33(2):62-68.

[34] Fukata $M$, Arditi $M$. The role of pattern recognition receptors in intestinal inflammation[J]. Mucosal Immunol. 2013, 6(3):451-63.

[35] Brint EK, MacSharry J, Fanning A, et al. Differential expression of toll-like 
receptors in patients with irritable bowel syndrome[J]. Am J Gastroenterol. 2011, 106(2):329-36.

[36] Sung J, Morales W, Kim G, et al. Effect of repeated Campylobacter jejuni infection on gut flora and mucosal defense in a rat model of post infectious functional and microbial bowel changes[J]. Neurogastroenterol Motil. 2013, 25(6):529-37.

[37] Belmonte L, Beutheu YS, Bertiaux-Vandaele N, et al. Role of toll like receptors in irritable bowel syndrome: differential mucosal immune activation according to the disease subtype[J]. PLoS One. 2012, 7(8):e42777.

[38] Haag LM, Fischer A, Otto B, et al. Campylobacter jejuni induces acute enterocolitis in gnotobiotic IL10-/- mice via Toll-like-receptor-2 and -4 signaling[J]. PLoS One. 2012, 7(7):e40761.

[39] Rakoff-Nahoum S, Paglino J, Eslami-Varzaneh F, et al. Recognition of commensal microflora by tolllike receptors is required for intestinal homeostasis[J]. Cell. 2004, 118(2):229-41.

[40] Chang ZL. Important aspects of Toll-like receptors, ligands and their signaling pathways[J]. Inflamm Res. 2010, 59(10):791-808.

[41] Karmarkar D, Rock KL. Microbiota signalling through MyD88 is necessary for a systemic neutrophilic inflammatory response[J]. Immunology. 2013, 140(4):483-92.

[42] Sanchez D, Rojas M, Hernandez I, et al. Role of TLR2- and TLR4-mediated signaling in Mycobacterium tuberculosis-induced macrophage death[J]. Cell Immunol.2010, 260(2):128-36.

[43]ZHANG Jie, LI Hong, WANG Jin-hai, SHAO Hui, LI Yong, YANG Long-bao.The effect and mechanism of intestinal microbiota in the pathogenesis of diarrhea-predominant irritable bowel syndrome[J]. World Clinical Drugs,2019,40(05):340-346.

[44]Li-Na Dong,Jun-Ping Wang,Ping Liu,Yun-Feng Yang,Jing Feng,Yi Han.Faecal and mucosal microbiota in patients with functional gastrointestinal disorders: Correlation with toll-like receptor 2/toll-like receptor 4 expression[J].World Journal of Gastroenterology,2017,23(36):6665-6673.

[45]Koçak Erdem,Akbal Erdem,Köklü Seyfettin,Ergül Bilal,Can Murat. The Colonic Tissue Levels of TLR2, TLR4 and Nitric Oxide in Patients with Irritable Bowel Syndrome[J]. Internal medicine (Tokyo, Japan),2016,55(9).

[46]CHAI Hong,WU Ya-ping,YANG Zheng,WU Cheng-yun,TAN Shi-yun,LUO He-sheng.The Effect sand Mechansm of Berberine on Irritable Bowel Syndrome Induced by Visceral Hypersen-sitivity and Abnormality in Rats[J].Chinese Journal of Microcirculation,2019,29(04):20-25.

[47]BIAN Zhao-xiang,ZHANG Gen-shui,HU Xu-guang,HUANG Jia-liang. Experimental study of traditional Chinese medicine compound CHFT and monomer components of paeoniflorin, tetrahydropalmatine and curcumin on hypersensitivity model rats[C]. China Academy of Chinese Medical Sciences; World 
Federation of Chinese Medicine Societies. Proceedings of the Third International Conference on Traditional Medicine. China Academy of Chinese Medical Sciences; World Federation of Chinese Medicine Societies: China Academy of Chinese Medical Sciences, 2004:322.

[48]SHUI Jie. Based on the 5-HT signaling pathway to study the mechanism of Tongxieyao Fang Baishao Fangfeng components to relieve IBS-D visceral hypersensitivity[D]. Chengdu University of Chinese Medicine, 2017.

\section{Figures}

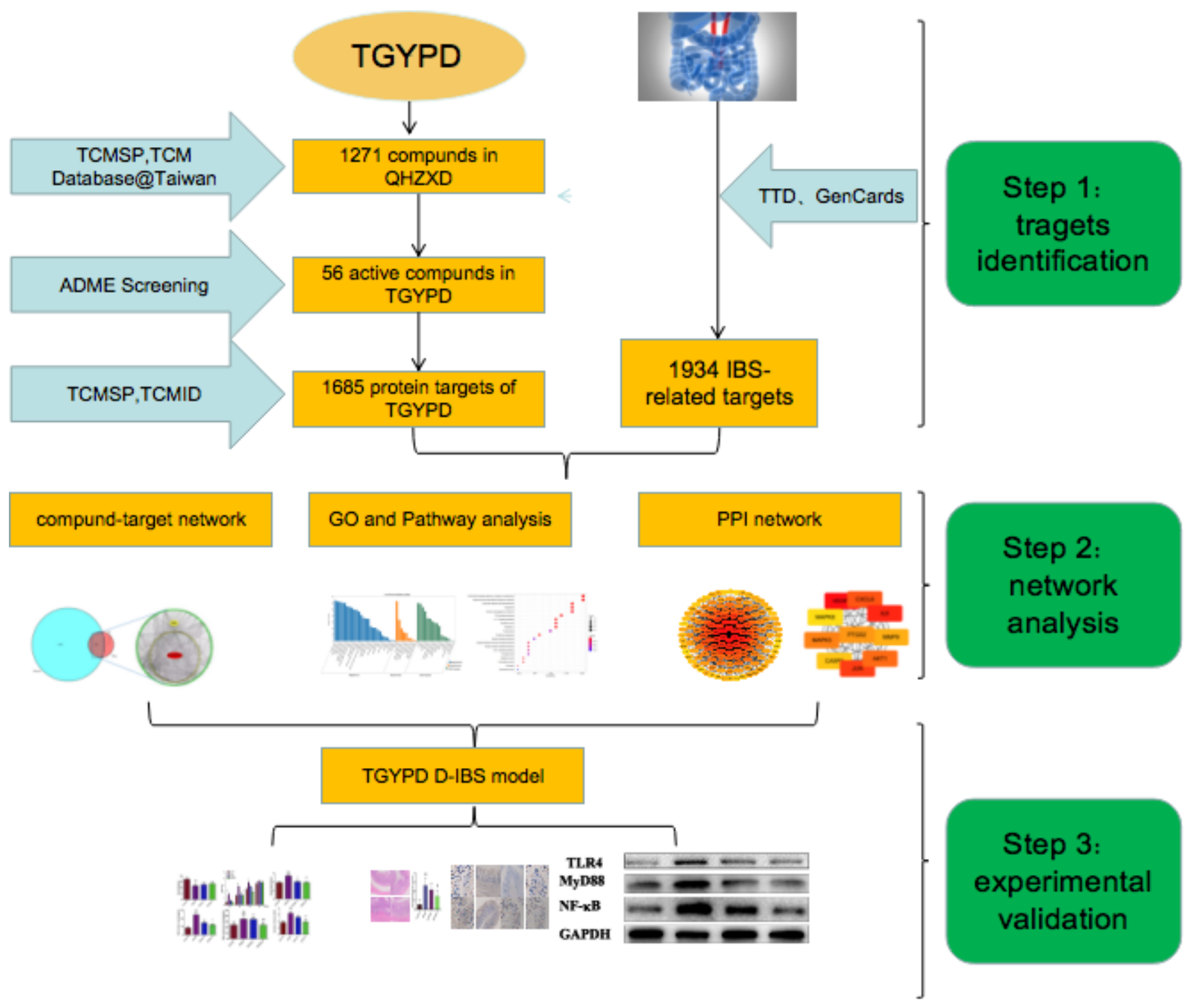

\section{Figure 1}

The technical strategy of the current study 


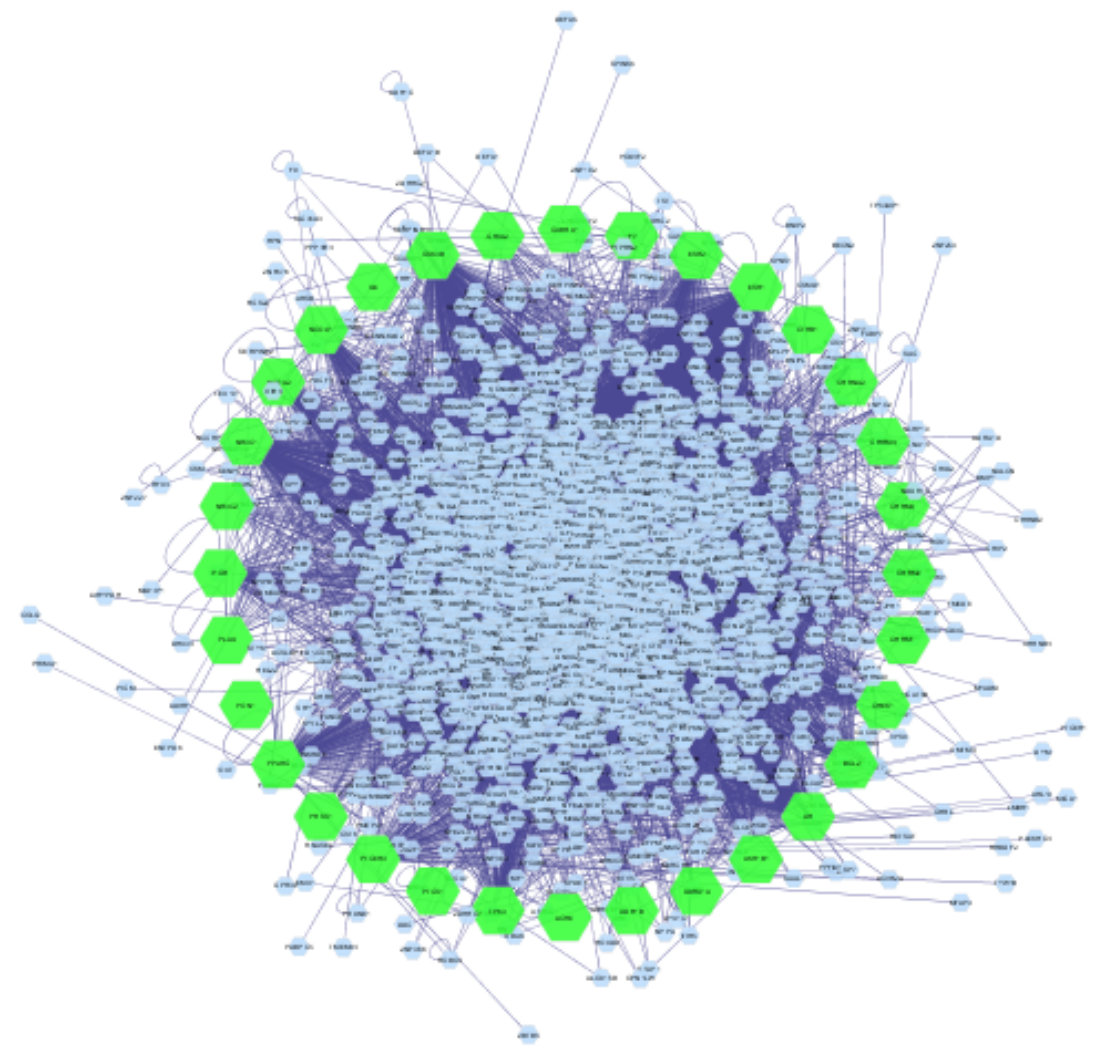

Figure 2

Network diagram of interactions among potential targets for Tiaogan Yunpi Decocotion

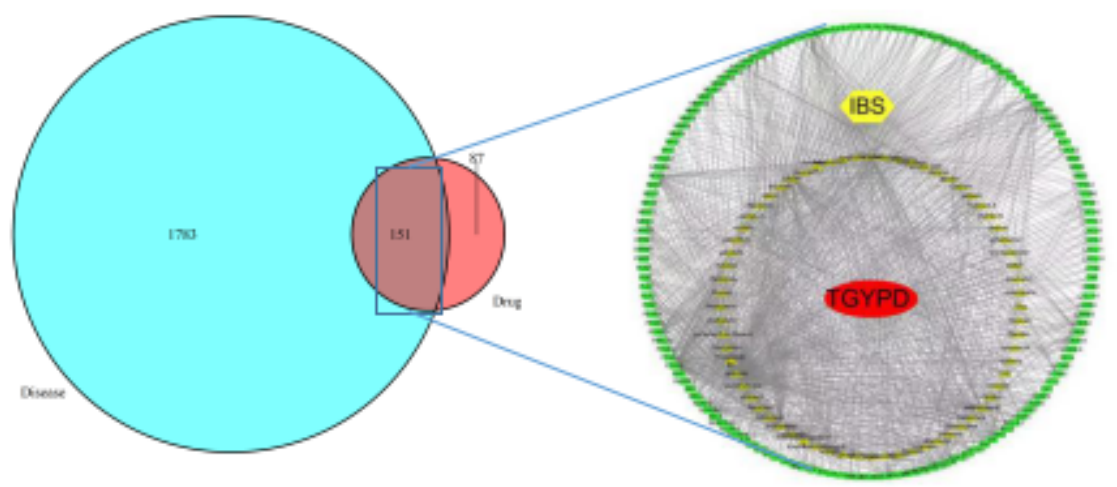

\section{Figure 3}

Drug-component-target network for active ingredients of Tiaogan Yunpi Decocotion and IBS 

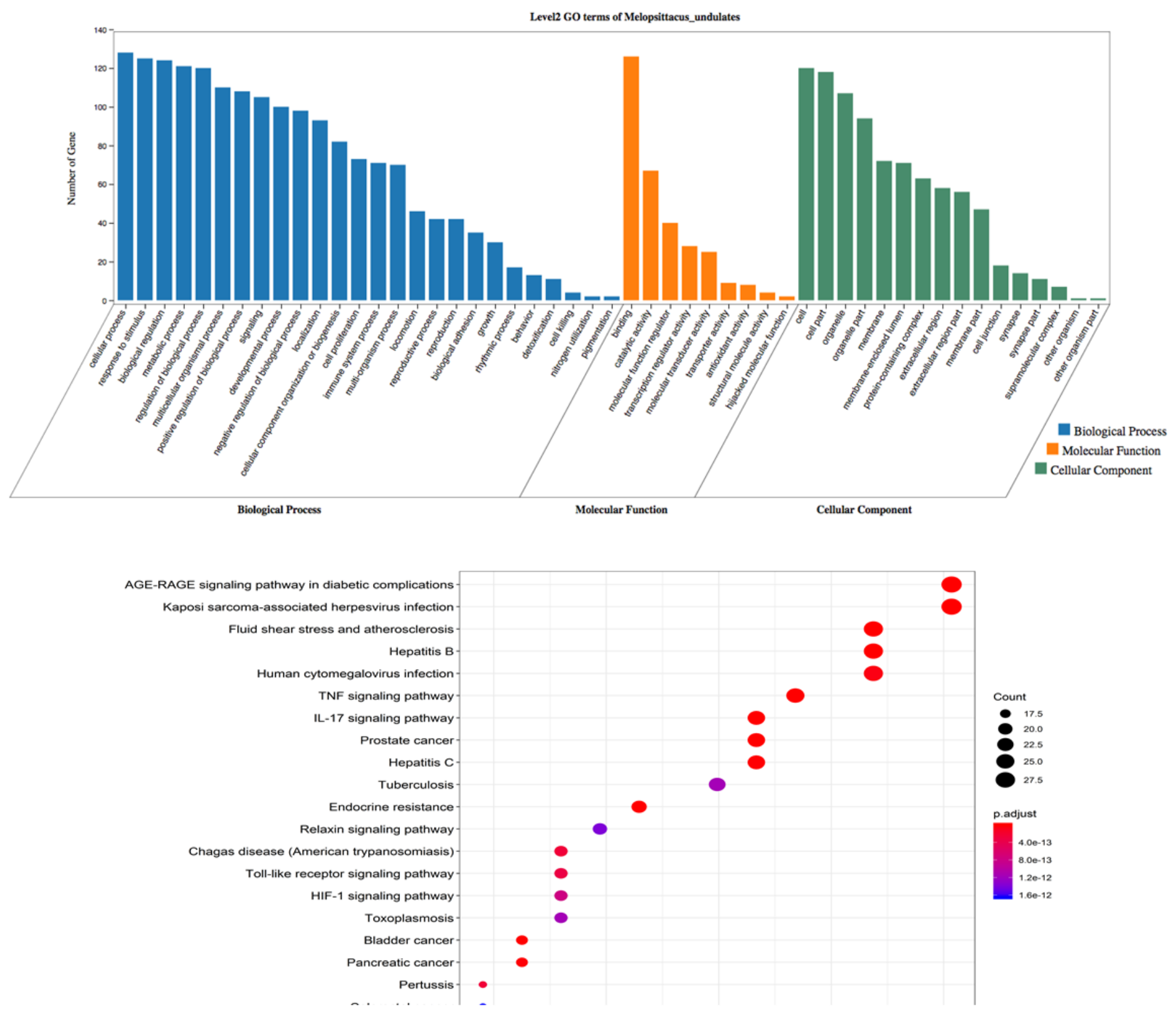

Figure 4

Enrichment analysis on GO biological processes(A) and KEGG pathway(B) of key targets from active compounds of Tiaogan Yunpi Decocotion 

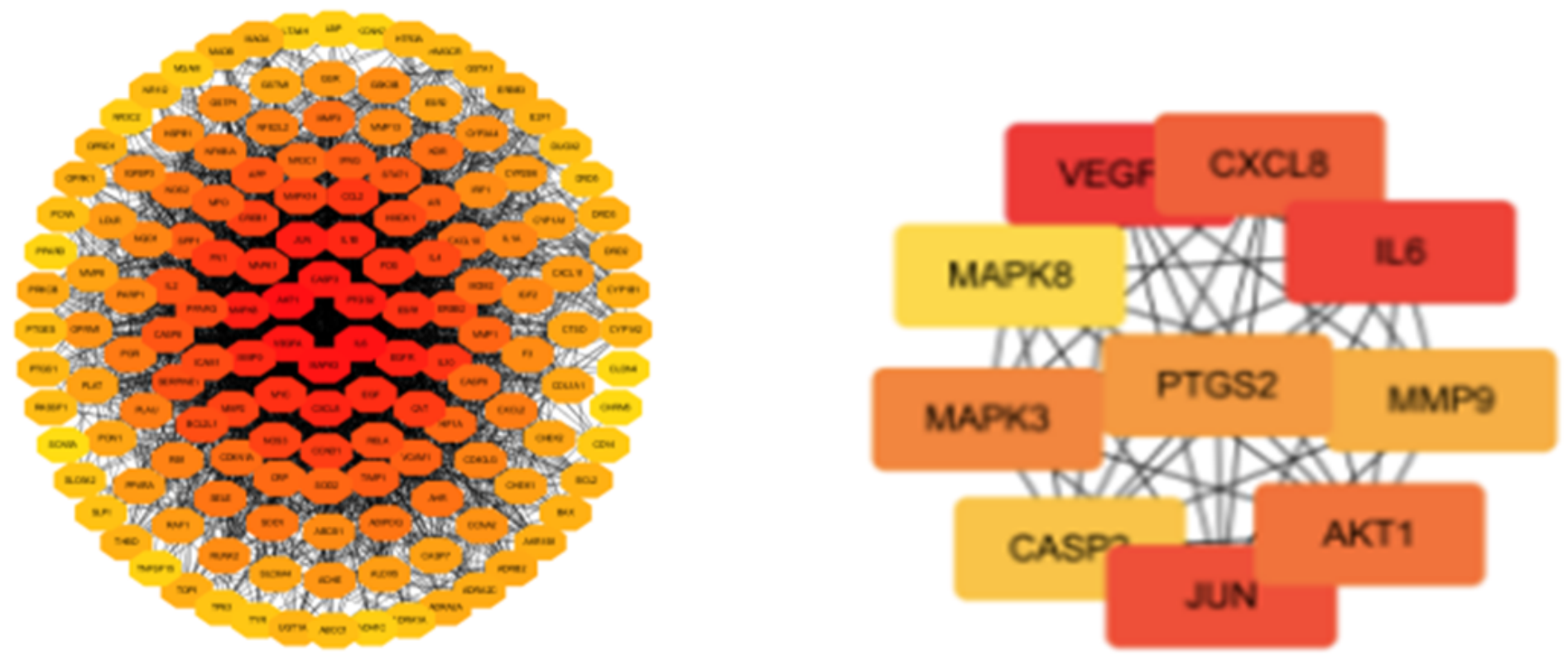

Figure 5

Protein interaction networks of anti-IBS genes in Tiaogan Yunpi Decocotion (A)and Subnetwork of the Hub gene(B)

A

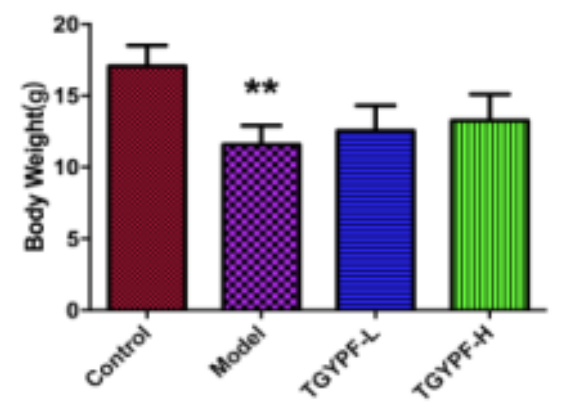

D
B

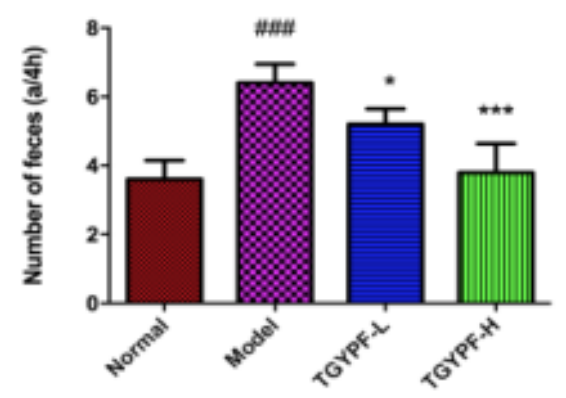

C

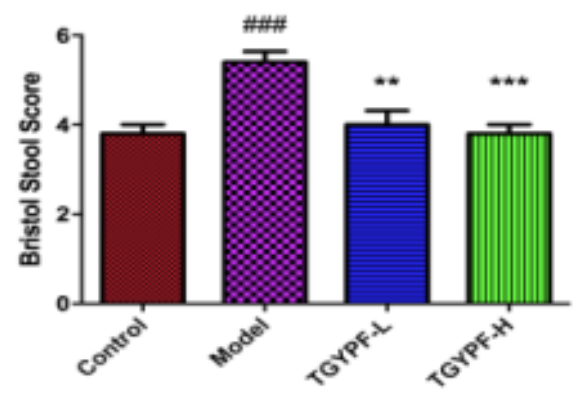

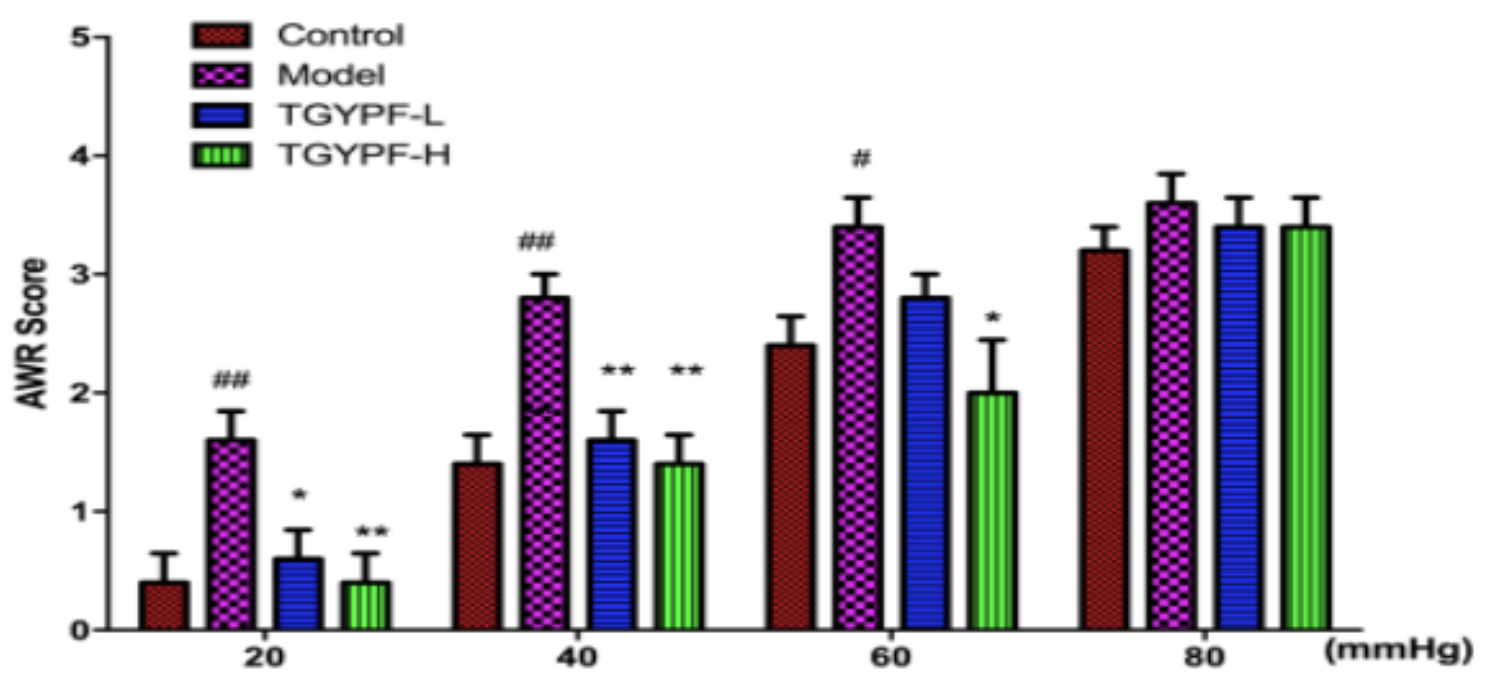

Figure 6 
Routine observation on D-IBS in rats. (A) Body weight; (B) Number of feces; (C) Bristol Stool Score;(D) AWR scores. Values were represented the mean \pm SEM. \#\#\#P<0.001,\#\#P<0.01, \#P<0.05 versus 5-FU group and ${ }^{* \star *} P<0.001,{ }^{* \star} P<0.01,{ }^{*} P<0.05$ versus normal group.

A

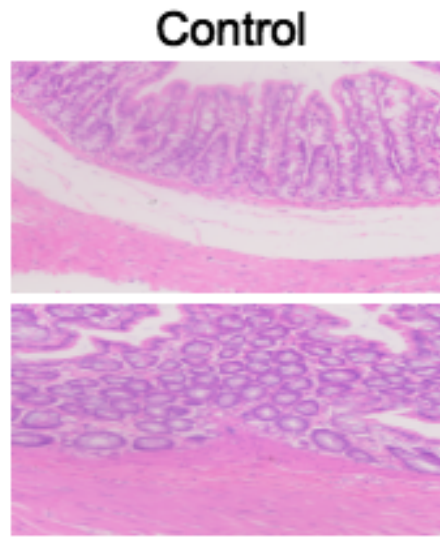

TGYPD-L

C

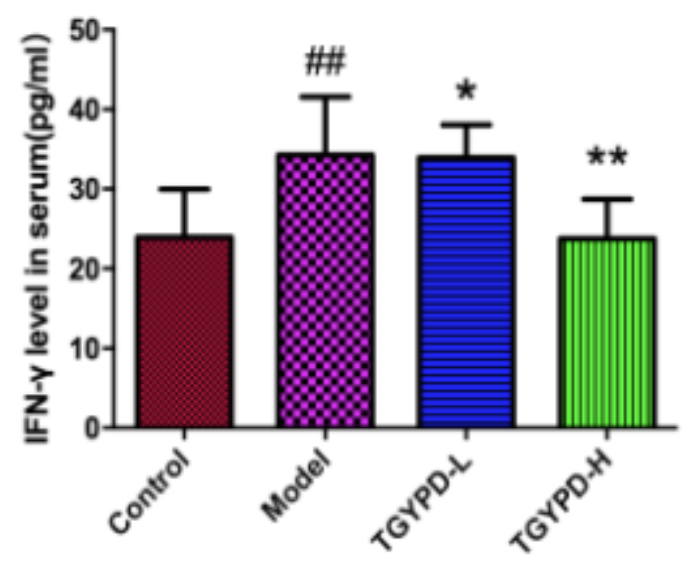

Model

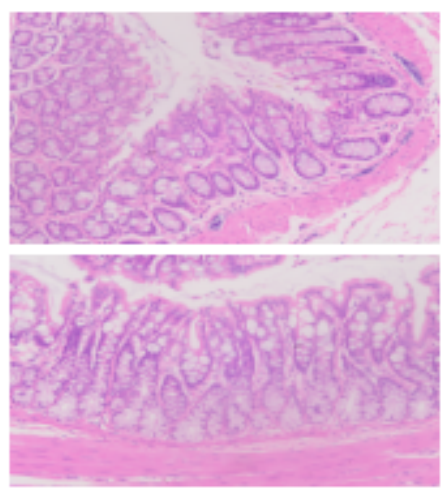

TGYPD-H
B

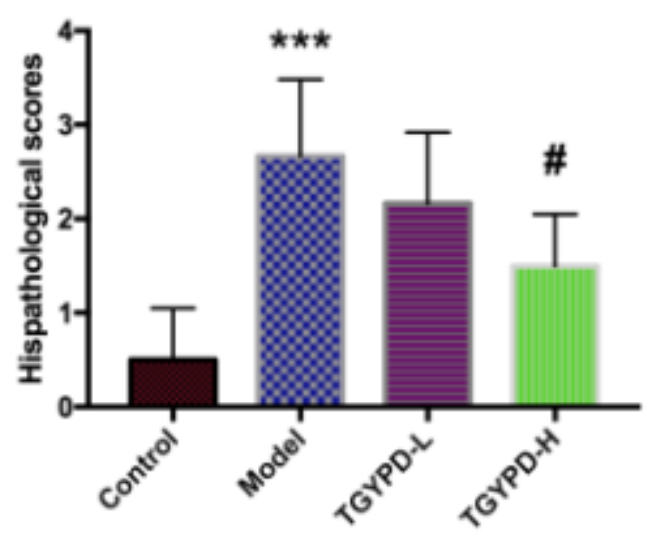

D

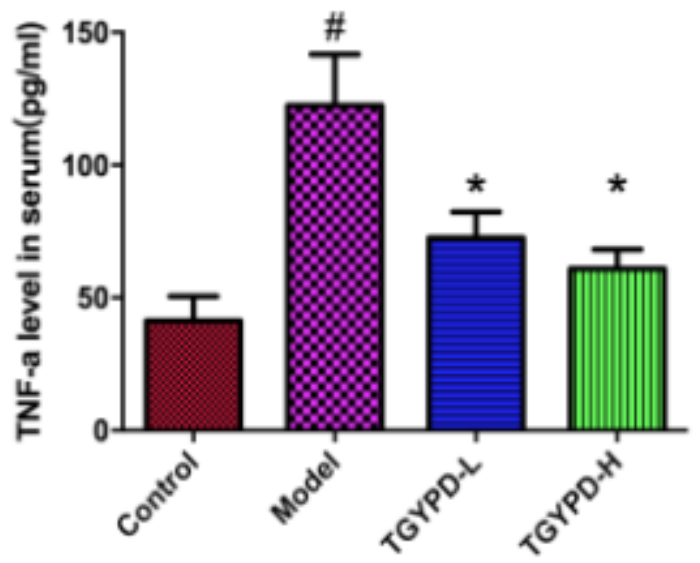

Figure 7

Histopathological assessment $(n=8,200 \times)$ and inflammtory cytokines $(n=8)$. (A)Red * labels and arrows indicate necrosis area, (B)Histopathological scores of colon. (C-D) Levels of TNF-a and IFN- $\gamma$. $\# \# \# P<0.001$,\#\#P $<0.01$, \#P $<0.05$ versus 5-FU group and ${ }^{\star \star \star *} P<0.001$ versus normal group. 
A

\section{TLR4 \\ MyD88 \\ NF-kB \\ GAPDH}

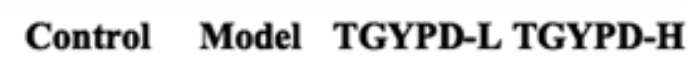

C

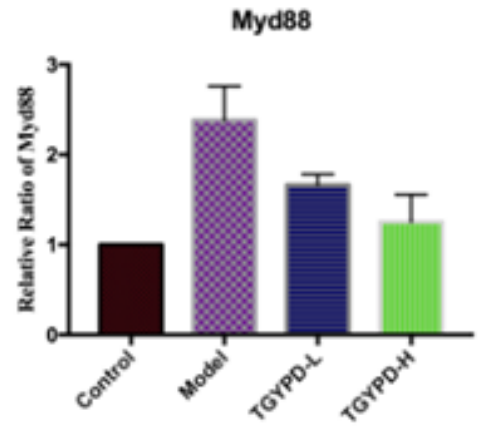

B

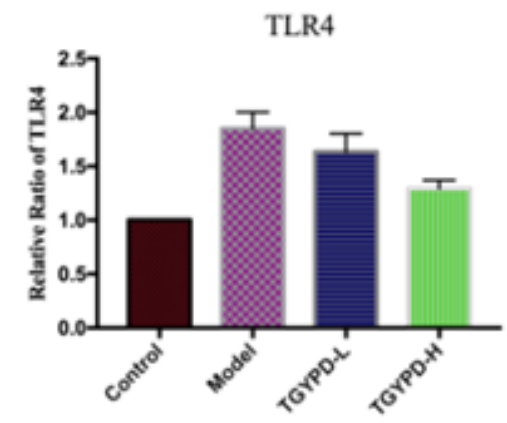

D

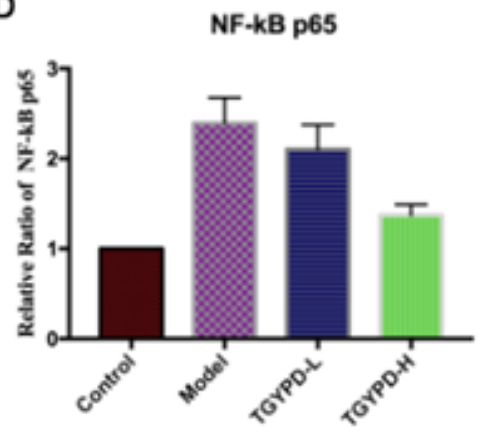

E

Control
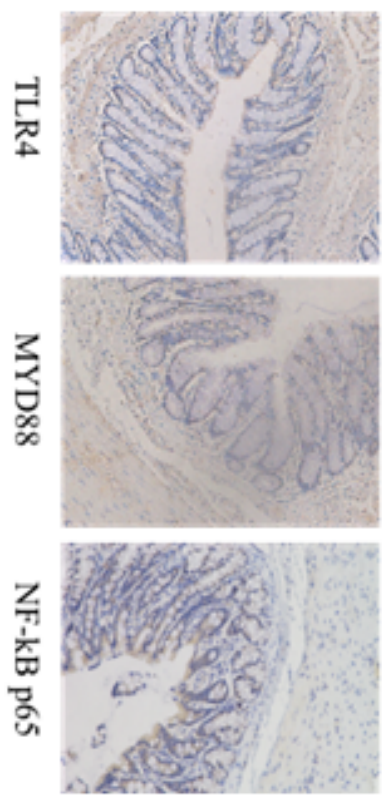

Model
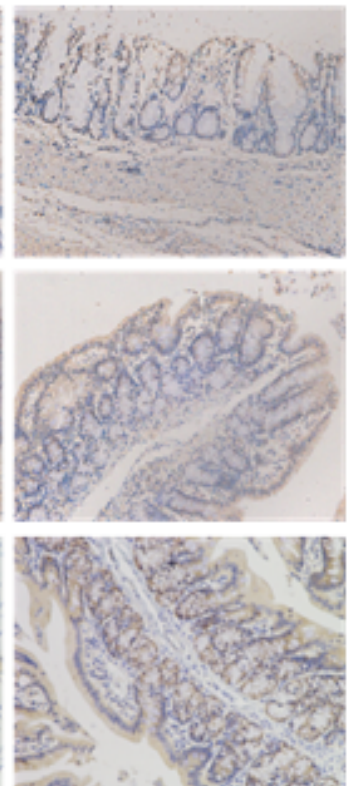

TGYPD-L
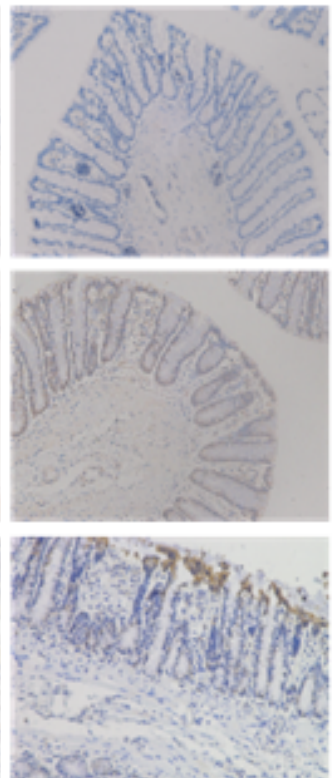

TGYPD-H
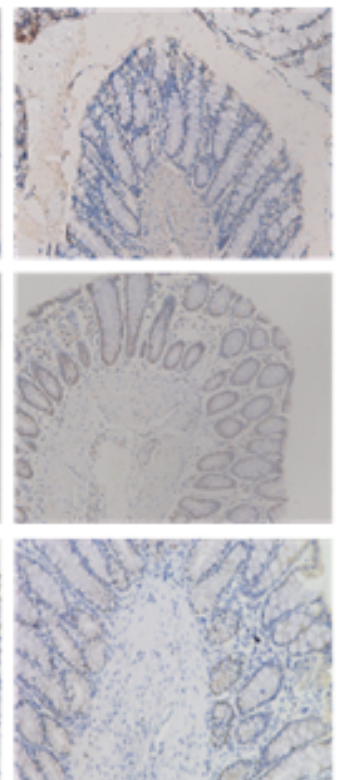

\section{Figure 8}

Effect of PA on TLR4/MyD88/NF-KB pathway proteins (A-D) and IHC staining (E,200 x), and positive results are purple spots and brown spots, respectively. Values were represented the mean \pm SEM. $* \star P<$ 0.01 , $\mathrm{P}<0.05$ versus 5 -FU group and \#\#P $<0.01$ versus normal group.

\section{Supplementary Files}


This is a list of supplementary files associated with this preprint. Click to download.

- .Supplement2.docx

- Supplement1.doc 